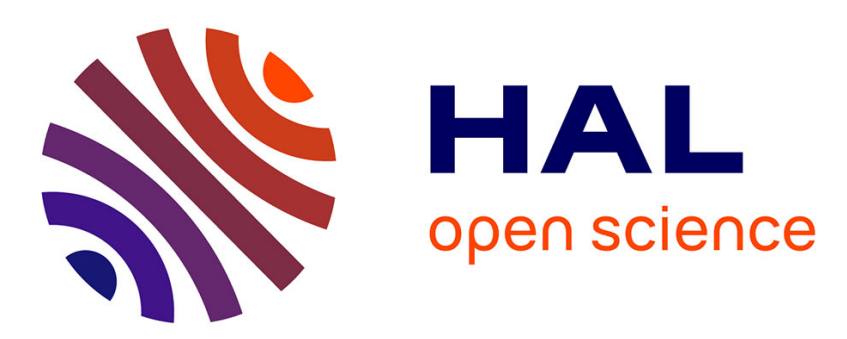

\title{
ALUTHGE OPERATOR FIELD AND ITS NUMERICAL RANGE AND SPECTRAL PROPERTIES
}

Gilles Cassier, Thomas Perrin

\section{- To cite this version:}

Gilles Cassier, Thomas Perrin. ALUTHGE OPERATOR FIELD AND ITS NUMERICAL RANGE AND SPECTRAL PROPERTIES. Integral Equations and Operator Theory, 2021, 93 (4), Article 41 (20 p.). 10.1007/s00020-021-02656-2 . hal-02995331

\section{HAL Id: hal-02995331 \\ https://hal.science/hal-02995331}

Submitted on 9 Nov 2020

HAL is a multi-disciplinary open access archive for the deposit and dissemination of scientific research documents, whether they are published or not. The documents may come from teaching and research institutions in France or abroad, or from public or private research centers.
L'archive ouverte pluridisciplinaire HAL, est destinée au dépôt et à la diffusion de documents scientifiques de niveau recherche, publiés ou non, émanant des établissements d'enseignement et de recherche français ou étrangers, des laboratoires publics ou privés. 


\title{
ALUTHGE OPERATOR FIELD AND ITS NUMERICAL RANGE AND SPECTRAL PROPERTIES
}

\author{
GILLES CASSIER $^{1 *}$ AND THOMAS PERRIN ${ }^{2}$
}

\begin{abstract}
For an arbitrary operator $T$ acting on a Hilbert space we consider a field of operators $\left(\Delta_{z}(T)\right)$ called the Aluthge operator field associated with $T$. After giving preliminary results, we establish that two fields (left and right), canonically linked to the Altuthge field $\left(\Delta_{z}(T)\right)$ and a support subspace, are constant on each horizontal segment where they are defined. This result leads to a positive solution of a conjecture stated by Jung-Ko-Pearcy in 2000. Then we do a detailed spectral study of $\left(\Delta_{z}(T)\right)$ and we give a Yamazaki type formula in this context.
\end{abstract}

\section{INTRODUCTION}

Let $H$ be a Hilbert space and let $B(H)$ be the algebra of all bounded operators acting on $H$. We write $\mathcal{K}(H)$ for the ideal of compact operators, $\mathcal{C}(H)$ for the quotient algebra $B(H) / \mathcal{K}(H)$ (called the Calkin algebra) and $\pi$ for the canonical surjection from $B(H)$ onto $\mathcal{C}(H)$. If $T \in B(H)$ we denote by $\sigma(T)$ the spectrum of $T$ and by $r(T)$ its spectral radius. Among familiar sets associated with $T$, recall that the numerical range of $T$ is defined as

$$
W(T)=\{\langle T x \mid x\rangle:\|x\|=1\} .
$$

As usual, we write $\sigma_{p}(T), \sigma_{a p p}(T), \sigma_{r e s}(T), \sigma_{e}(T), \sigma_{l e}(T), \sigma_{r e}(T)$ and $\sigma_{s u r j}(T)$ for the point spectrum, the approximate point spectrum, the residual spectrum, the essential spectrum, the left essential spectrum, the right essential spectrum and the surjective spectrum of $T$, respectively. Recall that an operator $T \in B(H)$ is said to be Weyl if it is Fredholm of index zero and Browder if it is Fredholm of finite ascent and descent. The Weyl spectrum $\sigma_{w}(T)$ and the Browder spectrum $\sigma_{b}(T)$ of $T \in B(H)$ are defined by

$$
\begin{aligned}
\sigma_{w}(T) & =\{\lambda \in \mathbb{C}: \lambda I-T \text { is not Weyl }\} \\
\sigma_{b}(T) & =\{\lambda \in \mathbb{C}: \lambda I-T \text { is not Browder }\} .
\end{aligned}
$$

Denote by $\mathcal{S}$ the open strip of the complex plane defined by

$$
\mathcal{S}=\{z \in \mathbb{C} ; 0<\Re(z)<1\} .
$$

Let $T \in B(H)$ and let $T=U|T|$ be its polar decomposition, we define the Aluthge field of operators associated with $T$ by setting

$$
\Delta_{z}(T)=|T|^{z} U|T|^{1-z}
$$

Date: $20 / 10 / 2020$.

* Corresponding author.

2020 Mathematics Subject Classification. Primary 47A12, 47A25, 47A65; Secondary 47A56.

Key words and phrases. Aluthge transform, numerical range, spectral properties. 
for any $z \in \overline{\mathcal{S}}$ (for $u$ such that $\Re(u) \geqslant 0$, the complex power $|T|^{u}$ of $|T|$ is defined bellow in Section 2). The operator $\Delta(T):=\Delta_{1 / 2}(T)$ is called the Aluthge transform of $T$ and was introduced in [1]. Since then, the Aluthge transformation has been studied by many authors in many areas of operator theory (see for example [2, 3, 4, 15, 7, 8, 9, 10, 12, 18, 20, 21, 22, 23, 24] and the references therein, the list is far to be exhaustive). In [1], A. Aluthge proved that if $T$ is $p$ hyponormal with $p \in[1 / 2,1$ [ then $\Delta(T)$ is hyponormal, and if $T$ is $p$-hyponormal with $p \in] 0,1 / 2[$ then $\Delta(T)$ is $(p+1 / 2)$-hyponormal, thus showing the regularizing effect of the Aluthge transformation. For $z=1$, the operator $\Delta_{1}(T)$ corresponds to the Duggal transform of $T$. For $\alpha \in] 0,1[$, it seems that the transformation $\Delta_{\alpha}: T \mapsto \Delta_{\alpha}(T)$ was firstly considered in [20].

In Section 2, we provide preliminary results which will be used throughout the paper. Some of them are of own interest (see for instance Theorem 2.5).

Section 3 is devoted to numerical range properties of the Aluthge field associated with an operator $T \in B(H)$. I. B. Jung, E. Ko and C. Pearcy (2000) have conjectured (conjecture 1.9 of [18]) that

$$
W(\Delta(T)) \subseteq W(T)
$$

for any $T \in B(H)$. In [22], P. Y. Wu has given the first result in this direction which is $\overline{W(\Delta(T))} \subseteq \overline{W(T)}$ (see also [23]). In the finite dimensional case, it gives (1.1). Later in [3], it is shown that $\overline{W\left(\Delta_{\alpha}(T)\right)} \subseteq \overline{W(T)}$ for each $\alpha \in[0,1]$. Notice that almost all results which are concerned with closure of numerical ranges are based on the following Hildebrandt's characterization of $\overline{W(T)}$ established in [16]

$$
\overline{W(T)}=\bigcap_{\zeta \in \mathbb{C}} D(\zeta,\|\zeta I-T\|] .
$$

The main result of this paper is Theorem 3.1 asserting that the two fields of operators (left and right), canonically associated with the Altuthge field $\left(\Delta_{z}(T)\right)$ and a support subspace (defined bellow in Subsection 2.6), are constant on each horizontal segment included in $\overline{\mathcal{S}}$. As a direct consequence, a positive answer to conjecture (1.1) is given. Even more, the inclusion

$$
W\left(\Delta_{z}(T)\right) \subseteq W(T)
$$

is obtained for each $z \in \overline{\mathcal{S}}$.

The last section deals with spectral properties of the Aluthge field $\left(\Delta_{z}(T)\right)_{z \in \overline{\mathcal{S}}}$ associated with an operator $T \in B(H)$. We carefully study different spectra of $\Delta_{z}(T)$, showing that most of them are preserved by the transformation $\Delta_{z}$ when $z \in \mathcal{S}$. Using counter examples we prove that some of these spectra are not preserved when $z$ belongs to the boundary of $\mathcal{S}$. Finally, by means of a short and direct proof, we show that a Yamazaki type formula holds for every $z \in \mathcal{S}$.

\section{Preliminaries}

Let $T \in B(H)$, as usually we write $\mathcal{N}(T)$ for the nullspace of $T, \mathcal{R}(T)$ for its range, $\operatorname{Lat}(T)$ for the set of invariant subspaces of $T$ and $\gamma(T)$ for the reduced minimum modulus of $T$. 
2.0.1. Power operator field associated with a positive operator. Let $R$ be a positive operator, using the spectral measure $E^{R}$ associated with $R$ we can define the power operator field $z \longrightarrow R^{z}$ defined on $\mathcal{P}_{+}=\{z \in \mathbb{C} ; \Re(z) \geqslant 0\}$ by setting

$$
R^{z}=\int_{] 0,\|R\|]} t^{z} d E^{R}(t) .
$$

The next proposition is a direct consequence of functional calculus associated with a positive operator and Lebesgue theorem.

Proposition 2.1. Let $R$ be a positive operator acting on a Hilbert space $H$. Then the operator field

$$
z \longrightarrow R^{z}
$$

is strongly continuous on the half plane $\mathcal{P}_{+}$, holomorphic on $\{\Re(z)>0\}$ and uniformly bounded on any closed strip of the form $\{z \in \mathbb{C} ; a \leqslant \Re(z) \leqslant b\}$ where $0 \leqslant a \leqslant b$.

Let $R \in B(H)$ be a positive operator, the following proposition summarizes a number of useful properties of the power operator field associated with $R$.

Proposition 2.2. Let $R$ be a nonzero positive operator acting on a Hilbert space H. Then, we have:

(i) $R^{0}=P_{\overline{\mathcal{R}(R)}}:=P$ and $R^{z_{1}+z_{2}}=R^{z_{1}} \circ R^{z_{2}}=R^{z_{2}} \circ R^{z_{1}}$ for any $\left(z_{1}, z_{2}\right) \in \mathcal{P}_{+}^{2}$;

(ii) $\mathcal{N}\left(R^{z}\right)=\mathcal{N}(R)$ for any $z \in \mathcal{P}_{+}$;

(iii) $\overline{\mathcal{R}\left(R^{z}\right)}=\overline{\mathcal{R}(R)}$ for any $z \in \mathcal{P}_{+}$;

(iv) $\gamma\left(R^{i t}\right)=1$ for any $t \in \mathbb{R}$ and $\gamma\left(R^{z}\right)=\gamma(R)^{2 \Re(z)}$ for each $z \in \mathcal{P}_{+} \backslash i \mathbb{R}$.

(v) Let $z_{0} \in \mathcal{P}_{+}$. Assume that $R^{z_{0}}$ is semi-Fredholm, then $R^{z_{0}}$ is Fredholm. Moreover, if $z_{0} \in \stackrel{\circ}{\mathcal{P}}_{+}$, then $R^{z}$ is Fredohlm for any $z \in \mathcal{P}_{+}$.

(vi) Let $\Omega$ be a Borelian subset of $\mathbb{R}$ with positive Lebesgue measure. Assume that $E \in \bigcap_{u \in i \Omega} \operatorname{Lat}\left(R^{u}\right)$, then $E \in \operatorname{Lat}\left(R^{z}\right)$ for any $z \in \stackrel{\circ}{\mathcal{P}}_{+}$.

Proof. (i) For any $z \in \mathcal{P}_{+}$and any $t \in[0,\|R\|]$, set $\varphi_{z}(t)=\mathbf{1}_{] 0,\|R\|]}(t) t^{z}\left(\varphi_{z}(0)=0\right)$. Notice that $\varphi_{0}(t)=\mathbf{1}_{] 0,\|R\|]}(t)$, hence $R^{0}=P_{\overline{\mathcal{R}(R)}}$. Since $\varphi_{z_{1}+z_{2}}=\varphi_{z_{1}} \times \varphi_{z_{1}}$, the equality $R^{z_{1}+z_{2}}=R^{z_{1}} \circ R^{z_{2}}=R^{z_{2}} \circ R^{z_{1}}$ follows from the Borelian functional calculus associated with $R$.

(ii) Let $x \in \mathcal{N}\left(R^{z}\right)$, then we have

$$
0=\int_{] 0,\|R\|]} \exp (2 \Re(z) \ln (t)) d E_{x, x}^{R}(t) .
$$

Therefore we have necessarily $\left.\left.E_{x, x}^{R}(] 0,\|R\|\right]\right)=0$ and then $\left.\left.E_{x, y}^{R}(] 0,\|R\|\right]\right)=0$ for any $y \in H$, so $R x=\left[\int_{0}^{\|R\|} t d E^{R}(t)\right] x=0$. Thus, $x \in \mathcal{N}(R)$. Conversely, if $x \in \mathcal{N}(R)$ we have $E_{x, y}^{R}=\langle x \mid y\rangle \delta_{0}$ for any $y \in H$. Then we get $\left\langle R^{z} x \mid y\right\rangle=$ $\int_{0}^{\|R\|} \varphi_{z}(t) d E_{x, y}^{R}=0$ for each $y \in H$ and hence $R^{z} x=0$. 
(iii) The equality $\overline{\mathcal{R}\left(R^{z}\right)}=\overline{\mathcal{R}(R)}$ follows from (ii).

(iv) Recall that the reduced modulus of an operator $T \in B(H)$ is defined by setting

$$
\gamma(T)=\inf \left\{\|T x\| ; x \in \mathcal{N}(T)^{\perp} \text { and }\|x\|=1\right\} .
$$

Let $z \in \mathcal{P}_{+} \backslash i \mathbb{R}$. On the one hand, from (i) we infer that $\left|R^{z}\right|^{2}=R^{\bar{z}} R^{z}=R^{2 \Re(z)}$ and hence $\left|R^{z}\right|=R^{\Re(z)}$. On the other hand, since $R$ is a positive operator, using Theorem 1.5 of [19], we see that $\gamma\left(R^{\Re(z)}\right)=\inf \left\{t ; t \in \sigma\left(R^{\Re(z)}\right) \backslash\{0\}\right\}=$ $\inf \left\{t^{\Re(z)} ; t \in \sigma(R) \backslash\{0\}\right\}=\inf \{t ; t \in \sigma(R) \backslash\{0\}\}^{\Re(z)}=\gamma(R)^{\Re(z)}$. Let $z=i t \in i \mathbb{R}$, then we have $\left|R^{i t}\right|=R^{0}=P(P \neq 0$ because $R \neq 0)$. It implies that $\gamma\left(R^{i t}\right)=\gamma\left(\left|R^{i t}\right|\right)=\gamma(P)=1$.

(v) Assume that $R^{z_{0}}$ is semi-Fredholm for some $z_{0} \in \mathcal{P}_{+}$, then the range of $R^{z_{0}}$ is closed and from (ii) we derive that $\operatorname{dim}\left(\mathcal{N}\left(R^{\overline{z_{0}}}\right)\right)=\operatorname{dim}\left(\mathcal{N}\left(R^{z_{0}}\right)\right)<+\infty$, so $R^{z_{0}}$ is Fredholm. Now suppose that $z_{0} \in \stackrel{\circ}{\mathcal{P}}_{+}$and let $z \in \mathcal{P}_{+}$. If $z \in i \mathbb{R}$, then the range of $R^{z}$ is closed. Thus, we may supposed that $z \in \stackrel{\circ}{\mathcal{P}}_{+}$. By (iv) we have

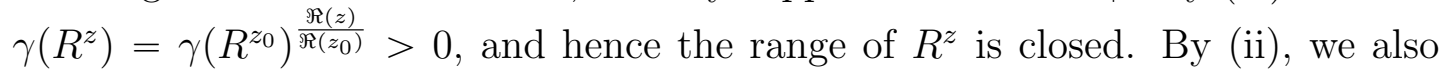
have $\operatorname{dim}\left(\mathcal{N}\left(R^{z}\right)\right)=\operatorname{dim}\left(\mathcal{N}\left(R^{\bar{z}}\right)\right)=\operatorname{dim}\left(\mathcal{N}\left(R^{\overline{z_{0}}}\right)\right)=\operatorname{dim}\left(\mathcal{N}\left(R^{z_{0}}\right)\right)<+\infty$. The conclusion follows.

(vi) Let $E \in \bigcap_{u \in i \Omega} \operatorname{Lat}\left(R^{u}\right)$ and $x, y$ be two vectors of $H$. Without loss of generality, we may suppose that $\|R\| \leqslant 1$. We introduce the function $f$ defined on the open unit disk $\mathbb{D}$ by $f(w)=\left\langle R^{\frac{1-w}{1+w}} x \mid y\right\rangle\left(\frac{1-w}{1+w} \in \stackrel{\circ}{\mathcal{P}}_{+}\right)$. Using Proposition 2.1. we see that $f$ is an analytic function. Besides, we have

$$
\left.\left.|f(z)| \leqslant \int_{] 0,1]}\left|r^{\frac{1-w}{1+w}}\right| d\left|E_{x, y}^{R}\right|(r) \leqslant \int_{] 0,1]} d\left|E_{x, y}^{R}\right|(r)=\left|E_{x, y}^{R}\right|(] 0,1\right]\right) \leqslant\|x\|\|y\| .
$$

Therefore, the function $f$ belongs to the space $H^{\infty}$ of bounded analytic functions on $\mathbb{D}$. Then, the function $f$ admits nontangential limits almost everywhere (see for instance [15]). Using again Proposition 2.1, we see that for any $w \in \mathbb{T} \backslash\{-1\}$, we still have $\lim _{r \rightarrow 1^{-}} f(r w)=\left\langle R^{\frac{1-w}{1+w}} x \mid y\right\rangle$. Let $z_{0} \in \stackrel{\circ}{\mathcal{P}}_{+}$and $w_{0}$ be such that $z_{0}=\frac{1-w_{0}}{1+w_{0}}$. Since $f \in H^{\infty}$ (see [15]), $f\left(w_{0}\right)$ is the Poisson integral of the boundary values of $f$. Then, we have

$$
\left\langle R^{z_{0}} x \mid y\right\rangle=\int_{]-\pi, \pi[}\left\langle R^{\frac{1-e^{i t}}{1+e^{i t}}} x \mid y\right\rangle P_{w_{0}}\left(e^{i t}\right) \frac{d t}{2 \pi} .
$$

Now, suppose that $x \in E$ and $y \in E^{\perp}$. Then $f(w)=0$ for any $w \in \tilde{\Omega}=$ $\left\{u \in \mathbb{T} \backslash\{-1\} ; \frac{1-u}{1+u} \in \Omega\right\}$. Clearly $\widetilde{\Omega}$ is of positive Lebesgue measure on the torus $\mathbb{T}$. As $f \in H^{\infty}$, we necessarily have $f\left(e^{i t}\right)=0$ for almost every $\left.t \in\right]-\pi, \pi$. From (2.1) we infer that $\left\langle R^{z_{0}} x \mid y\right\rangle=0$, thus $R^{z_{0}} x \in E$. Consequently $E \in \operatorname{Lat}\left(R^{z_{0}}\right)$. 
2.0.2. Ultrapower of a Hilbert space. Recall that a Banach limit $\mathcal{L}$ is a state on $l^{\infty}(\mathbb{C})$ which is shift invariant (that is $\left.\mathcal{L}\left(\left(u_{n+1}\right)_{n \geqslant 0}\right)=\mathcal{L}\left(\left(u_{n}\right)_{n \geqslant 0}\right)\right)$. If the sequence $\left(u_{n}\right)_{n \geqslant 0}$ converges to $l$, we have necessarily

$$
\mathcal{L}\left(\left(u_{n}\right)_{n \geqslant 0}\right)=l .
$$

Recall also that $0 \leqslant u_{n} \leqslant v_{n}$ implies $0 \leqslant \mathcal{L}\left(\left(u_{n}\right)_{n \geqslant 0}\right) \leqslant \mathcal{L}\left(\left(v_{n}\right)_{n \geqslant 0}\right)$. The associated ultrapower of a Hilbert space $H$ was firstly considered by S. K. Berberian in [6] and is constructed in the following way:

We consider the space $l^{\infty}(H)$ of bounded sequences $x=\left(x_{n}\right)$ of $H$, and define a positive sesquilinear form by setting

$$
(x \mid y)=\mathcal{L}\left(\left(\left\langle x_{n} \mid y_{n}\right\rangle\right)\right)
$$

and the corresponding Hilbertian seminorm

$$
n(x)=\sqrt{(x \mid y)}=\sqrt{\mathcal{L}\left(\left(\left\|x_{n}\right\|^{2}\right)\right)} .
$$

We set $\mathcal{N}=\left\{x \in l^{\infty}(H): n(x)=0\right\}$. The completion $\mathcal{H}:=\mathcal{H}_{\mathcal{L}}$ of $l^{\infty}(H) / \mathcal{N}$ is called the ultrapower of $H$ associated with $\mathcal{L}$. For any $x=\left(x_{n}\right) \in l^{\infty}(H)$, we denote by $\dot{x}=\dot{\left(x_{n}\right)}$ the associated point in $\mathcal{H}$. The map

$$
y \longmapsto \dot{y}=\dot{\overline{\left(y_{n}\right)}} \quad\left(\text { where } y_{n}=y \text { for all } \mathrm{n}\right)
$$

is a canonical isometric embedding of $H$ into $\mathcal{H}$. We consider the unital $\mathbf{C}^{*}$ morphism $\Phi$, from the von Neumann algebra $l^{\infty}(B(H))\left(l^{\infty}(B(H))\right.$ can be identify with the algebra of diagonal operators acting on $\left.l^{2}(H)=H \oplus \cdots \oplus H \oplus \cdots\right)$ into $B(\mathcal{H})$, which is defined by setting

$$
\Phi\left(\left(T_{n}\right)\right)(\dot{x})=\left(\widehat{T_{n}\left(x_{n}\right)}\right) .
$$

Notice that $\left\|\Phi\left(\left(T_{n}\right)\right)\right\| \leqslant \sup _{n \geqslant 0}\left\|T_{n}\right\|$. We set $\hat{T}=\Phi\left(\left(T_{n}\right)\right)$ where $T_{n}=T$ for each $n \in \mathbb{N}$. The map $\varphi: T \longmapsto \hat{T}$ is a $\mathbf{C}^{*}$-embedding of $B(H)$ into $B(\mathcal{H})$. The next Proposition gives some useful properties.

Proposition 2.3. Let $\left(T_{n}\right) \in l^{\infty}(B(H))$ and let $T \in B(H)$, then we have

(i) $\left\|\Phi\left(\left(T_{n}\right)\right)\right\| \in\left[\liminf \left\|T_{n}\right\|, \lim \sup \left\|T_{n}\right\|\right]$;

(ii) $\left|\Phi\left(\left(T_{n}\right)\right)\right|^{z}=\Phi\left(\left(\left|T_{n}\right|^{z}\right)\right)$ for any $z \in \mathcal{P}_{+}^{\circ}$;

(iii) $\Delta_{z}\left(\Phi\left(\left(T_{n}\right)\right)\right)=\Phi\left(\left(\Delta_{z}\left(T_{n}\right)\right)\right)$ for any $z \in \mathcal{S}$;

(iv) $\sigma(\hat{T})=\sigma(T)$;

(v) $\sigma_{p}(\hat{T})=\sigma_{\text {app }}(\hat{T})=\sigma_{\text {app }}(T)$;

(vi) Assume that the sequence $\left(\left\|T_{n}\right\|\right)_{n \geqslant 0}$ is convergent, then there exists $x=$ $\left(x_{n}\right) \in l^{\infty}(H)$ such that $\left|\Phi\left(\left(T_{n}\right)\right)\right|(\dot{x})=\left\|\Phi\left(\left(T_{n}\right)\right)\right\| \dot{x}$ with $\|\dot{x}\|=1$.

Proof. (i) For convenience we set $R=\Phi\left(\left(T_{n}\right)\right)$. Let $x=\left(x_{n}\right) \in l^{\infty}(H)$, then for any $p \in \mathbb{N}$ we have

$$
\left\|T_{n+p} x_{n+p}\right\|^{2} \leqslant\left[\sup _{n \geqslant p}\left\|T_{n}\right\|^{2}\right]\left\|x_{n+p}\right\|^{2},
$$

which implies $\|R \dot{x}\|^{2} \leqslant \sup _{n \geqslant p}\left\|T_{n}\right\|^{2}\|\dot{x}\|^{2}$ and hence $\|R\| \leqslant \sup _{n \geqslant p}\left\|T_{n}\right\|$. Letting $p$ goes to $+\infty$, we get $\|R\| \leqslant \lim \sup \left\|T_{n}\right\|$. Now, for each $n \in \mathbb{N}$ we choose a unit 
vector $x_{n}$ such that $\left\|T_{n} x_{n}\right\|^{2} \geqslant\left\|T_{n}\right\|^{2}-1 /(n+1)$ and consider $x=\left(x_{n}\right) \in l^{\infty}(H)$. Then, we have

$$
\|R\|^{2} \geqslant\|R \dot{x}\|^{2}=\mathcal{L}\left(\left(\left\|T_{n+p} x_{n+p}\right\|^{2}\right)\right) \geqslant \mathcal{L}\left(\left(\left\|T_{n+p}\right\|^{2}-\frac{1}{n+p+1}\right)\right) \geqslant \inf _{n \geqslant p}\left\|T_{n}\right\|^{2},
$$

and hence $\|R\| \geqslant \liminf \left\|T_{n}\right\|$.

(ii) Using the continuous functional calculus associated with positive operators, we see that it is a direct consequence of the fact that $\Phi$ is a continuous morphism which preserves the composition and the adjonction.

(iii) Let $\left(T_{n}\right) \in l^{\infty}(B(H))$. For each $n \in \mathbb{N}$, consider the polar decomposition $T_{n}=U_{n}\left|T_{n}\right|$. Set $A=\Phi\left(\left(T_{n}\right)\right), B=\Phi\left(\left(\Delta_{z}\left(T_{n}\right)\right)\right)$ and $U_{\Phi}=\Phi\left(\left(U_{n}\right)\right)$. Since $z \in \mathcal{S}$, we have $\Re(z)>0$ and $\Re(1-z)>0$ and hence $|A|^{u}=\Phi\left(\left(\left|T_{n}\right|^{u}\right)\right)$ for $u \in\{z, 1-z\}$ by (ii). It follows that $B=|A|^{z} U_{\Phi}|A|^{1-z}$. Let $A=U|A|$ be the polar decomposition of $A$. On the one hand, we have $\Delta_{z}(A)|A|=|A|{ }^{z} U|A||A|^{1-z}=$ $|A|^{z} U_{\Phi}|A||A|^{1-z}=B|A|$, so $\left.\Delta_{z}(A)\right|_{\overline{\mathcal{R}(|A|)}}=\left.B\right|_{\overline{\mathcal{R}(|A|)}}$. On the other hand, we have $0=\left.\Delta_{z}(A)\right|_{\mathcal{N}(|A|)}=\left.B\right|_{\mathcal{N}(|A|)}$ by using Proposition 2.2 (ii). Therefore, we get the desired equality $\Delta_{z}(A)=B=\Phi\left(\left(\Delta_{z}\left(T_{n}\right)\right)\right)$ for any $z \in \mathcal{S}$.

(iv) The inclusion $\rho(T) \subseteq \rho(\hat{T})$ is clear. For the reverse inclusion, consider the canonical isometric embedding of $H$ into $\mathcal{H}$. With this identification, we see that $H$ is a reducing subspace for $\left.\hat{T}\right|_{H}=T$ and hence $\rho(\hat{T}) \subseteq \rho(T)$.

(v) The equality $\sigma_{a p p}(\hat{T})=\sigma_{a p p}(T)$ follows directly from the fact that a complex number $\lambda$ does not belong to the approximate point spectrum $\sigma_{a p p}(A)$ of a operator $A$ if and only if there exists $\rho>0$ such that $\left(\bar{\lambda} I-A^{*}\right)(\lambda I-A) \geqslant \rho I$ and the fact that the application $T \longmapsto \hat{T}$ is a $\mathbf{C}^{*}$-embedding of $B(H)$ into $B(\mathcal{H})$. Taking into account the construction of $\mathcal{H}$, we easily see that $\sigma_{\text {app }}(T) \subseteq \sigma_{p}(\hat{T})$. Then, the equality $\sigma_{p}(\hat{T})=\sigma_{\text {app }}(\hat{T})$ comes from the previous equality $\sigma_{\text {app }}(\hat{T})=\sigma_{\text {app }}(T)$ and usual properties.

(vi) In this case, observe that (i) implies that $\left\|\Phi\left(\left(T_{n}\right)\right)\right\|=\lim \left\|T_{n}\right\|$. For each $n \in \mathbb{N}$, let $x_{n}$ be a unit vector such that $\left\|T_{n} x_{n}\right\|^{2} \geqslant\left\|T_{n}\right\|^{2}-1 /(n+1)$ and consider $x=\left(x_{n}\right) \in l^{\infty}(H)$. Then, we have $\|\dot{x}\|=1$ and

$$
\begin{aligned}
& \left\|\Phi\left(\left(T_{n}\right)\right)\right\|^{2} \geqslant\left\|\left|\Phi\left(\left(T_{n}\right)\right)\right| \dot{x}\right\|^{2}=\left\|\Phi\left(\left(\left|T_{n}\right|\right)\right) \dot{x}\right\|^{2}=\mathcal{L}\left(\left\|T_{n} x_{n}\right\|^{2}\right) \\
& \geqslant \mathcal{L}\left(\left\|T_{n}\right\|^{2}-\frac{1}{n+1}\right)=\lim \left\|T_{n}\right\|^{2}=\left\|\Phi\left(\left(T_{n}\right)\right)\right\|^{2}=\left\|\left|\Phi\left(\left(T_{n}\right)\right)\right|\right\|^{2} .
\end{aligned}
$$

Thus, we get $\left\|\left|\Phi\left(\left(T_{n}\right)\right)\right| \dot{x}\right\|=\left\|\left|\Phi\left(\left(T_{n}\right)\right)\right|\right\|$ which forces $\left|\Phi\left(\left(T_{n}\right)\right)\right| \dot{x}=\left\|\left|\Phi\left(\left(T_{n}\right)\right)\right|\right\| \dot{x}=$ $\left\|\Phi\left(\left(T_{n}\right)\right)\right\| \dot{x}$, since $\left|\Phi\left(\left(T_{n}\right)\right)\right|$ is a positive operator.

Remark 2.4. (1) In general, we do not have $\left\|\Phi\left(\left(T_{n}\right)\right)\right\|=\mathcal{L}\left(\left(\left\|T_{n}\right\|\right)\right)$. Indeed, set $T_{n}=I$ if $n$ is even and $T_{n}=0$ if $n$ is odd, then we see that $\Phi\left(\left(T_{n}\right)\right.$ is a non-zero orthogonal projection and hence $\left\|\Phi\left(\left(T_{n}\right)\right)\right\|=1$ while we have $\mathcal{L}\left(\left(\left\|T_{n}\right\|\right)\right)=1 / 2$.

(2) Consider a positive operator $A \in B(H)$ which is one-to-one but not invertible. 
Let $z=i t \in i \mathbb{R}$ and set $T_{n}=A$ for each $n \in \mathbb{N}$, then we easily check that $\Phi\left(\left(T_{n}^{i t}\right)\right)$ is a unitary operator, but $\Phi\left(\left(\left|T_{n}\right|\right)\right)^{i t}$ is not a unitary operator because $0 \in \sigma_{p}(|\hat{A}|)$ since $0 \in \sigma_{p}(\hat{A})=\sigma_{a p p}(\hat{A})=\sigma_{a p p}(A)$. Thus, Property (ii) cannot be extended to whole $\mathcal{P}_{+}$.

(3) By keeping the notations used in proof of (iii), we can remark that $U_{\Phi}$ may be different from $U$. Indeed, let us consider any one-to-one operator $T \in B(H)$ whose range is dense in $H$ but not equal to $H$, and set $T_{n}=T$ for each $n \in \mathbb{N}$. Then, the operators $U_{n}$ are all unitary and hence $U_{\Phi}$ is a unitary operator. But $U$ can not be a unitary operator since $0 \in \sigma_{p}(\hat{T})$. Thus, $\Phi$ does not preserve the polar decomposition.

The next result establishes some links between the iterated Aluthge fields associated with $T$ and the powers of $T$.

Theorem 2.5. Let $T \in B(H)$ be an operator of norm 1, then we have

(i) $\mathcal{N}\left(I-\left|T^{n+1}\right|\right)=\mathcal{N}\left(I-\left|\Delta_{z}^{n}(T)\right|\right)$ for each $z \in \mathcal{S}$;

(ii) Let $z \in \mathcal{S}$, then $\left\|\Delta_{z}^{n}(T)\right\|=1$ if and only if $\left\|T^{n+1}\right\|=1$.

Proof. (i) We proceed by induction. Denote by $T=U|T|$ the polar decomposition of $T$. For $n=0$, it is obvious. Assume that assertion (i) is true for some $n \in \mathbb{N}$. Let $x$ be a unit vector in $\mathcal{N}\left(I-\left|T^{n+2}\right|\right)$, then we have $1=\left\|T^{n+1} U|T| x\right\| \leqslant\||T| x\| \leqslant 1$. Thus, we get $1=\||T| x\|=\||T|\|$ which forces that $|T| x=x$, because $|T|$ is a positive operator. We infer that

$$
\begin{aligned}
1 & =\left\|T^{n+2} x\right\|=\left\|T^{n+1} U|T|^{1-z}|T|^{z} x\right\|=\left\|U|T|^{1-z} \Delta_{z}^{n+1}(T)|T|^{z} x\right\| \\
& =\left\|U|T|^{1-z} \Delta_{z}^{n+1}(T) x\right\| \leqslant\left\|\Delta_{z}(T)^{n+1} x\right\|=\left\|\left|\Delta_{z}(T)^{n+1}\right| x\right\| \leqslant\left\|\Delta_{z}(T)\right\| \leqslant 1 .
\end{aligned}
$$

Set $A=\Delta_{z}(T)$, then we see that $\|A\|=1$ and that $x \in \mathcal{N}\left(I-\left|A^{n+1}\right|\right)$. We can apply the induction assumption for $A$, this yields $x \in \mathcal{N}\left(I-\left|\Delta_{z}^{n}(A)\right|\right)=$ $\mathcal{N}\left(I-\left|\Delta_{z}^{n+1}(T)\right|\right)$. Conversely, assume that

$$
x \in \mathcal{N}\left(I-\left|\Delta_{z}^{n+1}(T)\right|\right)=\mathcal{N}\left(I-\left|\Delta_{z}^{n}(A)\right|\right) .
$$

By the induction assumption, it implies that $x \in \mathcal{N}\left(I-\left|A^{n+1}\right|\right)$, then we have $1=\left\|\left|A^{n+1}\right| x\right\|=\left\|A^{n+1} x\right\|=\left\|\Delta_{z}(T)^{n+2} x\right\|$. We write $z=a+i b$ with $\left.a \in\right] 0,1[$. We get $1=\left\|\Delta_{z}(T)^{n+1}|T|^{z} U|T|^{1-z} x\right\| \leqslant\left\||T|^{1-z} x\right\| \leqslant\left\||T|^{1-a} x\right\| \leqslant 1$. Since $|T|^{1-a}$ is a positive operator, it leads to $|T|^{1-a} x=x$. As $1-a>0$, we see that $\mathbb{C}\left[t^{1-a}\right]$ is dense in the space $C[0,1]$ of continuous functions on $[0,1]$. Then, the continuous functional calculus associated with $|T|$ ensures that $|T|^{u} x=x$ for any $u \in \mathcal{S}$. Then,

$$
\begin{aligned}
1 & =\left\|\Delta_{z}(T)^{n+1}|T|^{z} U|T|^{1-z} x\right\|=\left\||T|^{z} T^{n+1} U|T|^{1-z} x\right\| \\
& =\left\||T|^{z} T^{n+1} U|T| x\right\|=\left\||T|^{z} T^{n+2} x\right\| \leqslant\left\|T^{n+2} x\right\|=\left\|\left|T^{n+2}\right| x\right\| \leqslant 1,
\end{aligned}
$$

which implies that $x \in \mathcal{N}\left(I-\left|T^{n+2}\right|\right)$ and this completes the induction proof.

(ii) Assume that $\left\|\Delta_{z}^{n}(T)\right\|=1$. Using Proposition 2.3 ((i), (iii) and (v)), we obtain

$$
\left.1 \in \sigma_{\text {app }}\left(\left|\Delta_{z}^{n}(T)\right|\right)=\sigma_{p}\left(\varphi\left(\left|\Delta_{z}^{n}(T)\right|\right)\right)=\sigma_{p}\left(\left|\varphi\left(\Delta_{z}^{n}(T)\right)\right|\right)=\sigma_{p}\left(\left|\Delta_{z}^{n}(\hat{T})\right|\right)\right) .
$$


Hence, there exists a unit vector $\dot{x} \in \mathcal{H}$ which belongs to $\mathcal{N}\left(I-\left|\Delta_{z}^{n}(\hat{T})\right|\right)=$ $\mathcal{N}\left(I-\left|\hat{T}^{n+1}\right|\right)$ (by (i)). Therefore, we get $x=\left|\hat{T}^{n+1}\right| x$ and hence $1=\left\|\hat{T}^{n+1}\right\|=$ $\left\|T^{n+1}\right\|$. Using again (i) and Proposition 2.3, we prove in a similar way that the converse implication holds.

2.0.3. Support lines and support subspaces. Reccall that a infinite straight line $L$ in $\mathbb{C}$ may be written as : $\Re\left(e^{i \theta} z\right)=r$ with $(r, \theta) \in \mathbb{R}^{2}$. Let $C$ be a convex set in $\mathbb{C}$, we say that $L$ is a support line of $C$ if $\Re\left(e^{i \theta} z\right) \leqslant r$ for every $z \in C$ and $L \cap \partial C \neq \varnothing$. Let $T \in B(H)$, the well known theorem of Toeplitz and Hausdorff asserts that the numerical range $W(T)$ is convex. Therefore, we can consider support lines associated with $W(T)$ and observe that we have

Proposition 2.6. Let $T \in B(H)$ and $L$ a support line for $W(T)$ whose equation is $\Re\left(e^{i \theta} z\right)=r$, then the set of points $x \in H$ satisfying $\Re\left(e^{i \theta}\langle T x \mid x\rangle\right)=r\|x\|^{2}$ is a closed vectorial subspace of $H$.

Proof. Since $L$ is a support line for $W(T)$, we see that the operator $r I-\Re\left(e^{i \theta} T\right)$ is a positive operator and then admits a square root. Thus, we have

$$
\left\langle\left( r I-\Re\left(e^{i \theta} T\right) x|x\rangle=\left\|\sqrt{r I-\Re\left(e^{i \theta} T\right)} x\right\|^{2} .\right.\right.
$$

It easily implies that the set of points $x \in H$ satisfying $\Re\left(e^{i \theta}\langle T x \mid x\rangle\right)=r\|x\|^{2}$ is exactly $\mathcal{N}\left(r I-\Re\left(e^{i \theta} T\right)\right)$, and the conclusion follows.

Such subspaces will be called support subspaces for $T$.

\section{Aluthge field of operators and Numerical RANGe PRoperties}

The following result is due to the first author. Let $T \in B(H)$, the most striking fact is that the two fields (left and right), canonically associated with the Aluthge field $\left(\Delta_{z}(T)\right)_{z \in \overline{\mathcal{S}}}$ and a support subspace, are constant on each horizontal segment.

Theorem 3.1. Let $T \in B(H), z_{0} \in \mathcal{S}$ and $\lambda \in W\left(\Delta_{z_{0}}(T)\right) \cap \partial W(T)$, then there exists a support subspace $E$ for $\Delta_{z_{0}}(T)$ associated with a support line containing $\lambda$, not reduced to $\{0\}$, and such that the two operator fields $z \in \overline{\mathcal{S}} \longrightarrow P_{E} \Delta_{z}(T)$ and $z \in \overline{\mathcal{S}} \longrightarrow \Delta_{z}(T) P_{E}$ are constant on each horizontal segment contained in $\overline{\mathcal{S}}$. Moreover, if $T$ is one-to-one and has a dense range, then the operator $A:=$ $P_{E} \Delta_{z}(T) P_{E}$ is normal. In particular, we always have

$$
W\left(\Delta_{z}(T)\right) \subseteq W(T)
$$

for every $z \in \overline{\mathcal{S}}$ and any $T \in B(H)$.

Proof. Let $z=a+i b$ be in $\overline{\mathcal{S}}$ and $\lambda$ be in $W\left(\Delta_{z}(T)\right)$, then there exists a unit vector $x \in H$ such that $\lambda=\left\langle\Delta_{z}(T) x \mid x\right\rangle$. Using Proposition 2.1 and Proposition 2.2 , we can write

$$
\lambda=\left\langle\left.\Delta_{a}(T)|T|^{-i b} x|| T\right|^{-i b} x\right\rangle=\left\langle\Delta_{a}(T)\left[|T|^{-i b} x+Q x\right] \mid\left[|T|^{-i b} x+Q x\right]\right\rangle
$$

where $Q=P_{\mathcal{N}(T)}=I-P$. Observe that $\left\||T|^{-i b} x+Q x\right\|^{2}=\left\|P|T|^{-i b} P x+Q x\right\|^{2}=$ $\left\|P|T|^{-i b} P x\right\|^{2}+\|Q x\|^{2}=\left\|\left.T\right|^{-i b} P x\right\|^{2}+\|Q x\|^{2}=\|P x\|^{2}+\|Q x\|^{2}=\|x\|^{2}=1$. Therefore $\lambda \in W\left(\Delta_{a}(T)\right)$. Conversely, assume that $\lambda=\left\langle\Delta_{a}(T) x \mid x\right\rangle$, in a 
similar way we can see that $\lambda=\left\langle\Delta_{z}(T)\left[|T|^{i b} x+Q x\right] \mid\left[|T|^{i b} x+Q x\right]\right\rangle$ with $\left\||T|^{i b} x+Q x\right\|=1$, and hence $\lambda \in W\left(\Delta_{z}(T)\right)$. Consequently, for each $z \in \overline{\mathcal{S}}$, we have

$$
W\left(\Delta_{z}(T)\right)=W\left(\Delta_{\Re(z)}(T)\right) .
$$

From (3.1) and the fact that $\Delta_{z}(T)=|T|^{i \Im(z)} \Delta_{\Re(z)}(T)|T|^{-i \Im(z)}$, it suffices to prove Theorem 3.1 in the case where $\left.z_{0}=\alpha_{0} \in\right] 0,1[$.

On the one hand, since $W(T)$ is convex and $\lambda \in \partial W(T)$, we know that there exists a support line $L$ for $W(T)$ which contains $\lambda$. On the other hand, we have $\Delta_{\beta}\left(e^{i \theta} R\right)=e^{i \theta} \Delta_{\beta}(R)$ and $W\left(e^{i \theta} R\right)=e^{i \theta} W(R)$ for any triple $(R, \beta, \theta) \in$ $B(H) \times[0,1] \times \mathbb{R}$. Then, we are reduced to the case where there exists a unit vector $x_{0} \in H$ and a real number $r$ such that

$$
\forall z \in W(T) \quad \Re(z) \leqslant r, \lambda=\left\langle\Delta_{\alpha_{0}}(T) x_{0} \mid x_{0}\right\rangle \text { and } \Re(\lambda)=r .
$$

Since $W\left(\Delta_{\alpha}(T)\right) \subseteq \overline{W(T)}$ for any $\alpha \in[0,1], L$ is still a support line for $\Delta_{\alpha_{0}}(T)$ $\left(\lambda \in W\left(\Delta_{\alpha_{0}}(T)\right)\right)$. Let $E$ be the support subspace for $\Delta_{\alpha_{0}}(T)$ associated with $L$ (now $L$ is determined by the equation $\Re(z)=r$ ). Observe that $E$ is not reduced to $\{0\}$ because $x_{0} \in E$. Let $T=U|T|$ be the polar decomposition of $T$ and let $x$ be a unit vector in $H$, we consider the function $f$ defined on the closed strip $\overline{\mathcal{S}}$ by setting

$$
f(z)=\exp \left(\left\langle|T|^{z} U|T|^{1-z} x \mid x\right\rangle\right) .
$$

Using Proposition 4.2 we see that the two operator fields $z \longrightarrow|T|^{z}$ and $z \longrightarrow$ $|T|^{1-z}$ are strongly continuous on $\overline{\mathcal{S}}$. Since the strong convergence is preserved by products, it implies the strong continuity of the operator field $z \longrightarrow|T|^{z} U|T|^{1-z}$ and hence the continuity of $f$ on $\overline{\mathcal{S}}$. Besides, $f$ is analytic in $\mathcal{S}$.

Let $s \in \mathbb{R}$. On the one hand, since $\Re(T) \leqslant r I$ we have

$$
\begin{aligned}
|f(i s)| & =\exp \left(\left\langle\Re\left(|T|^{i s} U|T|^{1-i s}\right) x \mid x\right\rangle\right) \\
& =\exp \left(\left\langle\left.\Re(T)|T|^{-i s} x|| T\right|^{-i s} x\right\rangle\right) \leqslant \exp \left(r\left\||T|^{-i s} x\right\|^{2}\right) \leqslant \exp (r) .
\end{aligned}
$$

On the other hand, we get

$$
\begin{aligned}
|f(1-i s)| & =\exp \left(\left\langle\Re\left(|T|^{1-i s} U|T|^{i s}\right) x \mid x\right\rangle\right) \\
& =\exp \left(\left\langle\left.\Re(|T| U)|T|^{i s} x|| T\right|^{i s} x\right\rangle\right) \\
& =\exp \left(\left\langle\left. U^{*} \Re(T) U|T|^{i s} x|| T\right|^{i s} x\right\rangle\right) \leqslant \exp \left(r\left\|U|T|^{-i s} x\right\|^{2}\right) \leqslant \exp (r) .
\end{aligned}
$$

Then, the Hadamard three-lines theorem tells us that

$$
|f(z)| \leqslant(\exp (r))^{\Re(z)}(\exp (r))^{1-\Re(z)}=\exp (r)
$$

for any $z \in \overline{\mathcal{S}}$. This inequality leads to the next operatorial inequality

$$
\Re\left(|T|^{z} U|T|^{1-z}\right) \leqslant r I,
$$

valid for any $z \in \overline{\mathcal{S}}$. Let $x$ be a unit vector in $E$ and let $g$ be the function on $\overline{\mathcal{S}}$ given by $g(z)=\left\langle\Re\left(|T|^{z} U|T|^{1-z}\right) x \mid x\right\rangle$. As $x$ is a unit vector in $E$, we have $g\left(\alpha_{0}\right)=\left\langle\Re\left(\Delta_{\alpha_{0}}(T)\right) x \mid x\right\rangle=r$. Using (3.4), we derive that $g$ is a harmonic function in the open set $\mathcal{S}$ that is bounded above and attains its maximum at 
$\alpha_{0} \in \mathcal{S}$, therefore $g$ is constant in $\mathcal{S}$, and by continuity, in fact in all $\overline{\mathcal{S}}$. Thus, we get

$$
\Re\left(|T|^{z} U|T|^{1-z}\right) y=r y
$$

for every $y \in E$ and every $z \in \overline{\mathcal{S}}$.

Let $t$ be a real number and let $y \in E$, then 3.5 ensures that

$$
|T|^{-i t} \Re\left(\Delta_{\alpha_{0}}(T)\right)|T|^{i t} y=\Re\left(\Delta_{\alpha_{0}-i t}(T)\right) y=r y .
$$

As $|T|^{i t}|T|^{-i t}=P_{\overline{\mathcal{R}(|T|)}}$ and $\mathcal{R}\left(\Re\left(\Delta_{\alpha_{0}}(T)\right)\right) \subseteq \overline{\mathcal{R}(|T|)}$, we deduce that $|T|^{i t} y \in E$ for any $t \in \mathbb{R}$. Applying Proposition 2.2 (vi), we derive that $|T|$ is necessarily of the form $|T|=L_{1} \oplus L_{2}$ with respect the orthogonal sum $H=E \oplus E^{\perp}$, and where $L_{1}$ and $L_{2}$ are two positive operators. Let

$$
U=\left(\begin{array}{ll}
U_{1} & W \\
V & U_{2}
\end{array}\right)
$$

be the matrix representation relative to the direct sum $H=E \oplus E^{\perp}$ of the partial isometry $U$ associated with the polar decomposition $T=U|T|$. Then, we get

$$
\Re\left(\Delta_{\beta}(T)\right)=\frac{1}{2}\left(\begin{array}{ll}
L_{1}^{\beta} U_{1} L_{1}^{1-\beta}+L_{1}^{1-\beta} U_{1}^{*} L_{1}^{\beta} & L_{1}^{\beta} W L_{2}^{1-\beta}+L_{1}^{1-\beta} V^{*} L_{2}^{\beta} \\
L_{2}^{\beta} V L_{1}^{1-\beta}+L_{2}^{1-\beta} W^{*} L_{1}^{\beta} & L_{2}^{\beta} U_{2} L_{2}^{1-\beta}+L_{2}^{1-\beta} U_{2}^{*} L_{2}^{\beta}
\end{array}\right)
$$

for any $\beta \in[0,1]$. According to (3.5), we have $\Re\left(\Delta_{\beta}(T)\right) y=r y$ for any couple $(\beta, y) \in[0,1] \times E$. It forces $\Re\left(\Delta_{\beta}(T)\right)$ to be of the form

$$
\Re\left(\Delta_{\beta}(T)\right)=\left(\begin{array}{cc}
r I_{1} & 0 \\
0 & *
\end{array}\right) .
$$

Using (3.6) and (3.7), we obtain the following system of equations

$$
\left\{\begin{array}{l}
L_{1}^{\beta} U_{1} L_{1}^{1-\beta}+L_{1}^{1-\beta} U_{1}^{*} L_{1}^{\beta}=2 r I_{1} \\
L_{2}^{\beta} V L_{1}^{1-\beta}+L_{2}^{1-\beta} W^{*} L_{1}^{\beta}=0
\end{array}\right.
$$

for every $\beta \in[0,1]$. Let $\alpha \in[0,1]$, then by successively taking $\beta=\alpha$ and $\beta=1-\alpha \in[0,1]$ in the first equation of the system (3.8), we get the system of equations

$$
\left\{\begin{array}{l}
L_{1}^{\alpha} U_{1} L_{1}^{1-\alpha}+L_{1}^{1-\alpha} U_{1}^{*} L_{1}^{\alpha}=2 r I_{1} \\
L_{1}^{1-\alpha} U_{1} L_{1}^{\alpha}+L_{1}^{\alpha} U_{1}^{*} L_{1}^{1-\alpha}=2 r I_{1}
\end{array}\right.
$$

Summing the two equations of the system 3.9 we get

$$
L_{1}^{\alpha} \Re\left(U_{1}\right) L_{1}^{1-\alpha}+L_{1}^{1-\alpha} \Re\left(U_{1}\right) L_{1}^{\alpha}=2 r I_{1} .
$$

Set $A_{1}=\Re\left(U_{1}\right)$ and $P_{1}=P_{\overline{\mathcal{R}\left(L_{1}\right)}}$, and let $\tilde{A}_{1}=P_{1} A_{1} P_{1}$ and $\tilde{L}_{1}=P_{1} L_{1} P_{1}$ be the respective compressions of $A_{1}$ and $L_{1}$ that act on the subspace $\overline{\mathcal{R}\left(L_{1}\right)}$. Since the operators $P_{1}$ and $L_{1}$ commute, we derive from (3.10) that

$$
\begin{gathered}
2 r P_{1}=P_{1} L_{1}^{\alpha} P_{1} A_{1} P_{1} L_{1}^{1-\alpha} P_{1}+P_{1} L_{1}^{1-\alpha} P_{1} A_{1} P_{1} L_{1}^{\alpha} P_{1} \\
={\tilde{L_{1}}}^{\alpha} \tilde{A}_{1}{\tilde{L_{1}}}^{1-\alpha}+{\tilde{L_{1}}}^{1-\alpha} \tilde{A}_{1}{\tilde{L_{1}}}^{\alpha} .
\end{gathered}
$$

Then, taking $\alpha=0$ we obtain $2 r P_{1}=P_{1} \tilde{A}_{1} \tilde{L_{1}}+\tilde{L_{1}} \tilde{A}_{1} P_{1}$. Multiplying this last equality by $\tilde{L}_{1}$, once on the left and once on the right, we obtain $2 r \tilde{L}_{1} P_{1}=2 r \tilde{L}_{1}=$ $\tilde{L_{1}} P_{1} \tilde{A}_{1} \tilde{L}_{1}+{\tilde{L_{1}}}^{2} \tilde{A}_{1} P_{1}=\tilde{L_{1}}{\tilde{A_{1}}}_{\tilde{L}_{1}}+{\tilde{L_{1}}}^{2} \tilde{A}_{1}$ and $2 r \tilde{L_{1}}=P_{1}{\tilde{A_{1}}}_{\tilde{L}_{1}}{ }^{2}+\tilde{L}_{1} \tilde{A}_{1} P_{1} \tilde{L}_{1}=$ 
$P_{1} \tilde{A}_{1}{\tilde{L_{1}}}^{2}+\tilde{L_{1}} \tilde{A}_{1} \tilde{L}_{1}$, from which we deduce that ${\tilde{A_{1}}}_{\tilde{L}_{1}}{ }^{2}={\tilde{L_{1}}}^{2} \tilde{A}_{1}$. Then, using the continuous functional calculus associated with the positive operator $\tilde{L}_{1}$, we derive that

$$
\tilde{A}_{1} \tilde{L}_{1}=\tilde{L_{1}} \tilde{A}_{1} .
$$

Subtracting the two equations of the system (3.9), we obtain $L_{1}^{\alpha} \Im\left(U_{1}\right) L_{1}^{1-\alpha}+$ $L_{1}^{1-\alpha}\left(-\Im\left(U_{1}\right)\right) L_{1}^{\alpha}=0$. Set $A_{2}=\Im\left(U_{1}\right)$ and denote by $\tilde{A}_{2}$ the compression of $A_{2}$ to $\overline{\mathcal{R}\left(L_{1}\right)}$, we see that ${\tilde{L_{1}}}^{\alpha}{\tilde{A_{2}}}_{2}{\tilde{L_{1}}}^{1-\alpha}={\tilde{L_{1}}}^{1-\alpha}{\tilde{A_{2}}}_{\tilde{L}_{1}}{ }^{\alpha}$. Then, making $\alpha$ to be 0 we have $P_{1} \tilde{A}_{2} \tilde{L}_{1}=\tilde{L}_{1} \tilde{A}_{2} P_{1}$, and hence

$$
\tilde{A}_{2} \tilde{L}_{1}=\tilde{L}_{1} \tilde{A}_{2} .
$$

Taking into account (3.11) and (3.12), we finally obtain

$$
\tilde{U}_{1}{\tilde{L_{1}}}^{=}{\tilde{L_{1}}}_{U_{1}}
$$

where $\tilde{U}_{1}$ is the compression of $U_{1}$ to $\overline{\mathcal{R}\left(L_{1}\right)}$.

Now, we exploit the second equation of (3.8). Taking successively $\beta=\alpha$ and $\beta=1-\alpha$ in this equation, we deduce

$$
\left\{\begin{array}{l}
{\tilde{L_{2}}}^{\alpha} \tilde{V}{\tilde{L_{1}}}^{1-\alpha}+{\tilde{L_{2}}}^{1-\alpha} \tilde{W}^{*}{\tilde{L_{1}}}^{\alpha}=0 \\
{\tilde{L_{2}}}^{1-\alpha} \tilde{V}{\tilde{L_{1}}}^{\alpha}+{\tilde{L_{2}}}^{\alpha} \tilde{W}^{*}{\tilde{L_{1}}}^{1-\alpha}=0
\end{array}\right.
$$

where $P_{2}=P_{\overline{\mathcal{R}\left(L_{2}\right)}}, \tilde{L_{2}}=P_{2} L_{2} P_{2}, \tilde{V}=P_{2} V P_{1}$ and $\tilde{W}=P_{1} W P_{2}$. Set $C=$ $\tilde{V}+\tilde{W}^{*}$, by summing the two equations of the system $(3.14)$ we get ${\tilde{L_{2}}}^{\alpha} C{\tilde{L_{1}}}^{1-\alpha}+$ $\tilde{L}_{2}{ }^{1-\alpha} C \tilde{L}_{1}{ }^{\alpha}=0$. Thus we obtain

$$
C \tilde{L_{1}}=-\tilde{L_{2}} C
$$

by taking $\alpha=0$. Similarily, by subtraction, we get from 3.14

$$
D \tilde{L_{1}}=\tilde{L_{2}} D
$$

where $D=\tilde{V}-\tilde{W}^{*}$. Using (3.15) and (3.16), on the one hand we can see that $(D+C) \tilde{L_{1}}=\tilde{L_{2}}(D-C)$, and hence

$$
\tilde{V} \tilde{L}_{1}=-\tilde{L_{2}} \tilde{W}^{*} .
$$

On the other hand, we derive that $(D-C) \tilde{L_{1}}=\tilde{L_{2}}(D+C)$, and hence

$$
\tilde{L_{2}} \tilde{V}=-\tilde{W}^{*} \tilde{L}_{1} \text {. }
$$

Combining (3.17) and (3.17), we see that $\tilde{V_{1}}{\tilde{L_{1}}}^{2}=-{\tilde{L_{2}}}_{2} \tilde{W}^{*}{\tilde{L_{1}}}_{1}={\tilde{L_{2}}}^{2} \tilde{V}$ which implies $\tilde{V} p\left({\tilde{L_{1}}}^{2}\right)=p\left({\tilde{L_{1}}}^{2}\right) \tilde{V}$ for any complex polynomial $p$. Let $\left.\left.\beta \in\right] 0,1\right]$ and consider a sequence $\left(p_{n}\right)$ of complex polynomials converging to the function $\varphi$ in the space $C[0,\|T\|]$, where $\varphi$ is defined by setting $\varphi(t)=t^{\beta}$. Using the functional calculus associated with the two positive operators $\tilde{L}_{1}$ and $\tilde{L}_{2}$, we easily get

$$
\tilde{V}{\tilde{L_{1}}}^{\beta}={\tilde{L_{2}}}^{\beta} \tilde{V}
$$


We also observe that $\tilde{W}^{*}{\tilde{L_{1}}}^{2}=-\tilde{L_{2}} \tilde{V}{\tilde{L_{1}}}={\tilde{L_{2}}}^{2} \tilde{W}^{*}$. By working in the same way, we come to the second intertwining relation

$$
\tilde{W}^{*}{\tilde{L_{1}}}^{\beta}={\tilde{L_{2}}}^{\beta} \tilde{W}^{*}
$$

valid for any $\beta \in] 0,1]$. Let $\alpha \in] 0,1[$, by substituting the two intertwining relations (3.19) and (3.20) into the second equation of the system (3.14), we see that $0=$ ${\tilde{L_{2}}}^{1-\alpha} \tilde{V}{\tilde{L_{1}}}^{\alpha}+{\tilde{L_{2}}}^{\alpha} \tilde{W}^{*}{\tilde{L_{1}}}^{1-\alpha}={\tilde{L_{2}}}^{\alpha}{\tilde{L_{2}}}^{1-\alpha} \tilde{V}+{\tilde{L_{2}}}^{1-\alpha}{\tilde{L_{2}}}^{\alpha} \tilde{W}^{*}=\tilde{L_{2}}\left(\tilde{V}+\tilde{W}^{*}\right)$. Since by construction we have $\mathcal{R}\left(\tilde{V}+\tilde{W}^{*}\right) \subseteq \overline{\mathcal{R}\left(L_{2}\right)}$, we deduce that $\left(\tilde{V}+\tilde{W}^{*}\right) x \in$ $\mathcal{N}\left(L_{2}\right) \cap \overline{\mathcal{R}\left(L_{2}\right)}=\{0\}$ for any $x \in E$, and hence

$$
\tilde{W}=-\tilde{V}^{*} \text {. }
$$

Combining 3.13), 3.19), 3.20) and (3.21), we successively obtain

$$
\begin{aligned}
& L_{1}^{\alpha} U_{1} L_{1}^{1-\alpha}={\tilde{L_{1}}}^{\alpha} \tilde{U}_{1}{\tilde{L_{1}}}^{1-\alpha}=\tilde{U}_{1}{\tilde{L_{1}}}=P_{1} U_{1} L_{1}, \\
& L_{1}^{\alpha} W L_{2}^{1-\alpha}={\tilde{L_{1}}}^{\alpha} \tilde{W}{\tilde{L_{2}}}^{1-\alpha}={\tilde{L_{1}}}^{\alpha}{\tilde{L_{1}}}^{1-\alpha} \tilde{W} \\
& =\tilde{L_{1}} \tilde{W}=-\tilde{L_{1}} \tilde{V}^{*}=-L_{1} P_{1} V^{*} P_{2}=-L_{1} V^{*} P_{2} \text {, }
\end{aligned}
$$

From the above equalities, the strong continuity of $\left(\Delta_{z}(T)\right)_{z \in \overline{\mathcal{S}}}$ and the fact that $\Delta_{z}(T)=|T|^{i \Im(z)} \Delta_{\Re(z)}(T)|T|^{-i \Im(z)}$, we obtain that the two operator fields $z \in$ $\overline{\mathcal{S}} \longrightarrow P_{E} \Delta_{z}(T)$ and $z \in \overline{\mathcal{S}} \longrightarrow \Delta_{z}(T) P_{E}$ are constant on each horizontal segment contained in $\overline{\mathcal{S}}$.

Now, assume that $T$ is one-to one and has a dense range. Then, the operator $U$ in the polar decomposition of $T$ is unitary. Therefore, using (3.21) we derive that $I_{1}=U_{1} U_{1}^{*}+W W^{*}=U_{1} U_{1}^{*}+V^{*} V$, and that $I_{1}=U_{1}^{*} U_{1}+V^{*} V$. Thus $U_{1}$ is normal (in a similar way, we can prove that $U_{2}$ is also normal). In this case $U_{1}=\tilde{U}_{1}$ commutes with $L_{1}=\tilde{L_{1}}$, and then $A=P_{1} U_{1} L_{1}=U_{1} L_{1}$ is normal.

Let $T \in B(H)$, the last step in the proof consists in showing that

$$
W\left(\Delta_{z}(T)\right) \subseteq W(T)
$$

for every $z \in \overline{\mathcal{S}}$. Taking into account $(3.1)$, we are reduced to prove this inclusion for $z=\alpha_{0} \in[0,1]$. When $\alpha_{0} \in\{0,1\}$, we easily check this inclusion. Thus, we may assume that $\left.\alpha_{0} \in\right] 0,1\left[\right.$. Since $W\left(\Delta_{\alpha}(T)\right) \subseteq \overline{W(T)}$, we can suppose that $\lambda \in W\left(\Delta_{\alpha_{0}}(T)\right) \cap \partial W(T)$. In this case we have shown that there exists a unit vector $w$ such that $\lambda=\left\langle\Delta_{\alpha_{0}}(T) w \mid w\right\rangle$, and a support subspace $E$ containing $w$ such that the operator field $\left(\Delta_{\alpha}(T) P_{E}\right)_{\alpha \in[0,1]}$ is constant. We derive that

$$
\lambda=\left\langle\Delta_{\alpha_{0}}(T) w \mid w\right\rangle=\left\langle\Delta_{\alpha_{0}}(T) P_{E} w \mid w\right\rangle=\left\langle\Delta_{0}(T) P_{E} w \mid w\right\rangle=\left\langle P_{\overline{\mathcal{R}(|T|)}} T w \mid w\right\rangle .
$$

Two possibilities occurs:

- Firstly, if $T$ is one-to-one, then $P_{\overline{\mathcal{R}(|T|)}}=I$ and there is nothing to do.

- Secondly, if $T$ is not one-to-one, then $0 \in W(T)$. So, we may assume that $\lambda \neq 0$ and hence $P_{\overline{\mathcal{R}(|T|)}} w \neq 0$. We can write

$$
\lambda=\left(1-\left\|P_{\overline{\mathcal{R}(|T|)}} w\right\|^{2}\right) \times 0+\left\|P_{\overline{\mathcal{R}(|T|)}} w\right\|^{2}\left\langle T\left(\frac{P_{\overline{\mathcal{R}(|T|)}} w}{\left\|P_{\overline{\mathcal{R}(|T|)}} w\right\|}\right) \mid \frac{P_{\overline{\mathcal{R}(|T|)}} w}{\| P_{\overline{\mathcal{R}(|T|)}} w}\right\rangle,
$$


and then the Toeplitz-Hausdorff theorem tells us that $\lambda \in W(T)$. This achieves the proof of Theorem 3.1.

Remark 3.2. (1) For $\alpha=\frac{1}{2}$, the inclusion $W(\Delta(T)) \subseteq W(T)$ given by Theorem 3.1 answers positively to Conjecture 1.9 of [18].

(2) Assume that $T$ is one-to-one and has a dense range. Let $E$ be a support subspace for $\Delta_{z}(T)(z \in \mathcal{S})$ wich is associated with a complex scalar $\lambda$ and $U$ be the partial isometry given by the polar decomposition of $T$. Then, we have seen in the proof of Theorem 3.1 that $U$ takes the form

$$
U=\left(\begin{array}{cc}
U_{1} & * \\
* & U_{2}
\end{array}\right),
$$

with respect to the orthogonal decomposition $H=E \oplus E^{\perp}$, where the two operators $U_{1}$ and $U_{2}$ are normal.

\section{Spectral Properties for Aluthge operator fields}

4.0.1. Different type of spectra and Aluthge operator fields. Some spectral results are known for $\Delta_{\frac{1}{2}}(T):=\Delta(T)$ where $T \in B(H)$ (see for instance [17], and Theorem 1.3 and Theorem 1.5 of [18]). In the next theorem, we extend a number of useful spectral properties of $\Delta(T)$ to the Aluthge operator field $\left(\Delta_{z}(T)\right)_{z \in \overline{\mathcal{S}}}$ and establish some new spectral properties in this context.

Theorem 4.1. Let $T$ be an operator acting on a Hilbert space $H$. Then the Aluthge operator field associated with $T$ satisfies the following properties:

(i) We have $\sigma_{\text {surj }}\left(\Delta_{z}(T)\right) \backslash\{0\}=\sigma_{\text {surj }}(T) \backslash\{0\}$ and $\sigma_{\text {surj }}(T) \subseteq \sigma_{\text {surj }}\left(\Delta_{z}(T)\right)$ for any $z \in \overline{\mathcal{S}}$. The last inclusion may be strict.

(ii) $\sigma\left(\Delta_{z}(T)\right)=\sigma(T)$ for any $z \in \overline{\mathcal{S}}$;

(iii) (a) For any $z \in \overline{\mathcal{S}}$ we have $\sigma_{e}\left(\Delta_{z}(T)\right)=\sigma_{e}(T)$;

(b) Let $z \in \overline{\mathcal{S}} \backslash[1+i \mathbb{R}]$ then we have $\sigma_{l e}\left(\Delta_{z}(T)\right)=\sigma_{l e}(T)$. When $z \in 1+i \mathbb{R}$, we have $\sigma_{l e}\left(\Delta_{z}(T)\right) \subseteq \sigma_{l e}(T)$ and the inclusion may be strict;

(c) For any $z \in \overline{\mathcal{S}}$, we have $\sigma_{r e}(T) \subseteq \sigma_{r e}\left(\Delta_{z}(T)\right)$. Moreover the inclusion may be strict for all $z \in \overline{\mathcal{S}}$;

(iv) $\sigma_{w}\left(\Delta_{z}(T)\right)=\sigma_{w}(T)$ for any $z \in \overline{\mathcal{S}}$;

(v) $\sigma_{b}\left(\Delta_{z}(T)\right)=\sigma_{b}(T)$ for any $z \in \overline{\mathcal{S}}$;

(vi) $\sigma_{p}\left(\Delta_{z}(T)\right)=\sigma_{p}(T)$ for any $z \in \overline{\mathcal{S}}$;

(vii) $\sigma_{\text {app }}\left(\Delta_{z}(T)\right)=\sigma_{\text {app }}(T)$ for any $z \in \overline{\mathcal{S}} \backslash[1+i \mathbb{R}]$. If $z \in 1+i \mathbb{R}$, we have $\sigma_{\text {app }}\left(\Delta_{z}(T)\right) \subseteq \sigma_{\text {app }}(T)$ and the inclusion may be strict;

(viii) $\sigma_{\text {res }}\left(\Delta_{z}(T)\right)=\sigma_{\text {res }}(T)$ for each $z \in i \mathbb{R}$ and $\sigma_{\text {res }}\left(\Delta_{z}(T)\right) \subseteq \sigma_{\text {res }}(T)$ for any $z \in \overline{\mathcal{S}} \backslash[i \mathbb{R}]$. Moreover, in this latter case, the inclusion may be strict.

Proof. (i) Let $A, B$ be two operators acting on $H$ and let $\lambda$ be a nonzero complex number such that $\lambda I-A B$ is surjective. Then $\lambda I-A B$ admits a right inverse $X \in B(H)$. Set $Y=\frac{1}{\lambda}[I+B X A]$, then we have $(\lambda I-B A) Y=$ $\frac{1}{\lambda}[\lambda I-B A+B(\lambda I-A B) X A]=\frac{1}{\lambda}[\lambda I-B A+B A]=I$, thus $\lambda I-A B$ admits a right inverse. As the operators $A$ and $B$ play a symetric role, we deduce that $\sigma_{\text {surj }}(A B) \backslash\{0\}=\sigma_{\text {surj }}(B A) \backslash\{0\}$. Then, using Proposition 2.1 (i), we see 
that $\sigma_{\text {surj }}\left(\Delta_{z}(T)\right) \backslash\{0\}=\sigma_{\text {surj }}(T) \backslash\{0\}$. Now, suppose that $\Delta_{z}(T)$ is onto, then it implies that $|T|^{z}$ is onto and that $|T|^{\bar{z}}$ is one-to-one. By Proposition 2.2 (ii), we have $\{0\}=\mathcal{N}\left(|T|^{z}\right)=\mathcal{N}\left(|T|^{z}\right)$, we finally get that $|T|^{z}$ is invertible. Thus $T=\left(|T|^{z}\right)^{-1} \Delta_{z}(T)|T|^{z}$ is onto. Taking into account the two previous properties we get $\sigma_{\text {surj }}(T) \subseteq \sigma_{\text {surj }}\left(\Delta_{z}(T)\right)$. Let $T$ be a pure coisometry, then we have $0 \notin \sigma_{\text {surj }}(T)$. As in [7] (Proposition 4), we can see that $\left.\Delta_{z}(T)\right)=T^{*} T^{2}$ which is clearly not surjective and hence $0 \in \sigma_{\text {surj }}\left(\Delta_{z}(T)\right)$. Therefore, the inclusion $\sigma_{\text {surj }}(T) \subseteq \sigma_{\text {surj }}\left(\Delta_{z}(T)\right)$ may be strict.

(ii) Since $\sigma\left(|T|^{z} U|T|^{1-z}\right) \backslash\{0\}=\sigma\left(U|T|^{1-z}|T|^{z}\right) \backslash\{0\}=\sigma(T) \backslash\{0\}$, it remains to prove that $T$ is invertible if and only if $\Delta_{z}(T)$ is invertible. Assume that $T$ is invertible, then we successively see that $|T|$ and $|T|^{z}$ are invertible, hence $\Delta_{z}(T)=\left(|T|^{z}\right)^{-1} T|T|^{z}$ is invertible. Conversely, assume that $\Delta_{z}(T)$ is invertible, then there exists $X \in B(H)$ such that $|T|^{z} U|T|^{1-z} X=X|T|^{z} U|T|^{1-z}=I$, it implies that $|T|^{z}$ is onto and $\{0\}=\mathcal{N}\left(|T|^{\overline{1-z}}\right)=\mathcal{N}\left(|T|^{\bar{z}}\right)$, thus $|T|^{z}$ is invertible and hence $T=|T|^{z} \Delta_{z}(T)\left(|T|^{z}\right)^{-1}$ is invertible.

(iii) Let $\lambda \in \mathbb{C}^{*}$ and suppose that there exists $X$ such that $X(\lambda I-A B)=I+K_{1}$ (resp. $\left.(\lambda I-A B) X=I+K_{2}\right)$, where $K_{1}, K_{2}$ belong to the space $\mathcal{K}(H)$ of all compact operators on $H$. As in the proof of (i), set $Y=\frac{1}{\lambda}[I+B X A]$, then we easily check that $Y(\lambda I-B A)=I+\frac{1}{\lambda} B K_{1} A\left(\operatorname{resp} .(\lambda I-B A) Y=I+\frac{1}{\lambda} B K_{2} A\right)$ with $\frac{1}{\lambda} B K_{i} A \in \mathcal{K}(H)(i \in\{1,2\})$. It follows that $\sigma_{e}\left(\Delta_{z}(T)\right) \backslash\{0\}=\sigma_{e}(T) \backslash\{0\}$, $\sigma_{l e}\left(\Delta_{z}(T)\right) \backslash\{0\}=\sigma_{l e}(T) \backslash\{0\}$ and $\sigma_{r e}\left(\Delta_{z}(T)\right) \backslash\{0\}=\sigma_{r e}(T) \backslash\{0\}$. Consequently, To prove (a) (b) and (c), it suffices to examine what happens at the point 0.

(a) In the sequel, we denote by $\mathcal{F}$ (resp. $\mathcal{S} \mathcal{F}$ ) the class of Fredholm (resp. semiFrdohlm) operators in $B(H)$. Assume that $0 \in \rho_{e}(T)$, then $T \in \mathcal{F}$ and hence $|T| \in$ $\mathcal{F}$ which implies in turn that $U \in \mathcal{F}$. By Proposition $2.2(\mathrm{v})$, we see that $|T|^{u} \in \mathcal{F}$ for any $u \in \overline{\mathcal{S}}$. Therefore $\pi\left(\Delta_{z}(T)\right)=\pi\left(|T|^{z}\right) \pi(U) \pi\left(|T|^{1-z}\right)$ is invertible in $\mathcal{C}(H)$ as a product of elements of $G L(\mathcal{C}(H))$, equivalently $0 \in \rho_{e}\left(\Delta_{z}(T)\right)$. Conversely, suppose that $0 \in \rho_{e}\left(\Delta_{z}(T)\right)$, then we derive that $|T|^{z}$ and $|T|^{1-z}$ are both in $\mathcal{S F}$. Since $\Re(z)$ and $\Re(1-z)$ are not both zero, using Proposition 1 (v) we see that $|T|^{u} \in \mathcal{F}$ for any $u \in \overline{\mathcal{S}}$, and hence $\pi(U)=\pi\left(|T|^{z}\right)^{-1} \pi\left(\Delta_{z}(T)\right) \pi\left(|T|^{1-z}\right)^{-1} \in$ $G L(\mathcal{C}(H))$. Thus $\pi(T)=\pi(U) \pi(|T|) \in G L(\mathcal{C}(H))$ which gives that $0 \in \rho_{e}(T)$.

(b) Assume that $0 \in \rho_{l e}(T)$, then $|T| \in \mathcal{S} \mathcal{F}$ which implies, as we have seen before, that $|T|^{u} \in \mathcal{F}$ for any $u \in \overline{\mathcal{S}}$. Thus, $\pi(U)$ is left invertible in $\mathcal{C}(H)$ and hence $0 \in \rho_{l e}\left(\Delta_{z}(T)\right)$ for any $z \in \overline{\mathcal{S}}$. Now, let $z \in \overline{\mathcal{S}} \backslash[1+i \mathbb{R}]$ and suppose that $0 \in \rho_{l e}\left(\Delta_{z}(T)\right)$, it implies that $|T|^{1-z}$ is in $\mathcal{S F}$. As $\Re(1-z) \neq 0$, we get that $|T|^{u} \in \mathcal{F}$ for any $u \in \overline{\mathcal{S}}$ and hence $U \in \mathcal{S} \mathcal{F}$. We derive that $0 \in \rho_{l e}(T)$. Summarizing, we obtain $\sigma_{l e}\left(\Delta_{z}(T)\right)=\sigma_{l e}(T)$ for each $z \in z \in \overline{\mathcal{S}} \backslash[1+i \mathbb{R}]$ and $\sigma_{l e}\left(\Delta_{z}(T)\right) \subseteq \sigma_{l e}(T)$ when $z \in 1+i \mathbb{R}$.

Let $\left(e_{n}\right)_{n \geqslant 0}$ be the canonical orthonormal basis of the Hilbert space $H:=l^{2}(\mathbb{N})$. One may then define operators $A$ and $B$ in $B(H)$ by setting $A e_{n}=\frac{1}{2^{n}} e_{2 n+1}$ and $B e_{n}=e_{2 n}$. We consider the operator $T$ acting on $\mathcal{H}=H \oplus H$ which is given by 
the $2 \times 2$ matrix

$$
T=\left(\begin{array}{ll}
0 & 0 \\
A & B
\end{array}\right) .
$$

We can check that $|A| e_{n}=\frac{1}{2^{n}} e_{n}$ and that $A^{*} B=0$. Notice also that $B$ is an isometry, then we get

$$
|T|=\left(\begin{array}{cc}
|A| & 0 \\
0 & I
\end{array}\right)
$$

Let $T=U|T|$ be the polar decomposition of $T$. Since $\mathcal{R}(A) \cap \mathcal{R}(B)=\{0\}$ and $A$ is one-to-one, we derive that $T$ is one-to-one, and hence $U$ is an isometry. Taking into account that $U$ is the unique isometry $W$ such that $T=W|T|$, we see that $U$ is necessarily given by

$$
U=\left(\begin{array}{cc}
0 & 0 \\
V & B
\end{array}\right)
$$

where $V$ is the partial isometry (here $V$ is an isometry) associated with the polar decomposition of $A=V|A|$. Then, for any $z \in 1+i \mathbb{R}$ we obtain

$$
\Delta_{z}(T)=\left(\begin{array}{cc}
0 & 0 \\
V|A|^{-i \Im(z)} & B
\end{array}\right) .
$$

On the one hand, we see that $\Delta_{z}(T)$ is an isometry and hence $0 \notin \sigma_{l e}\left(\Delta_{z}(T)\right)$. On the other hand, let $Q$ be the orthogonal projection on the subspace $H \oplus\{0\}$, then we get

$$
T Q=\left(\begin{array}{ll}
0 & 0 \\
A & 0
\end{array}\right) \in \mathcal{K}(\mathcal{H})
$$

Then, from Theorem 1.1 of [1] we deduce that $0 \in \sigma_{l e}(T)$. Thus the inclusion $\sigma_{l e}\left(\Delta_{z}(T)\right) \subseteq \sigma_{l e}(T)$ is strict for each $z \in 1+i \mathbb{R}$.

(c) Let $z \in \overline{\mathcal{S}}$ and suppose that $0 \in \rho_{\text {re }}\left(\Delta_{z}(T)\right)$. We infer that $\pi\left(|T|^{z}\right)$ is right invertible in $\mathcal{C}(H)$ and hence invertible (Proposition $2.2(\mathrm{v})$ ). It successively implies that $\pi\left(U|T|^{1-z}\right)$ and $\pi(T)=\pi\left(U|T|^{1-z}\right) \pi\left(|T|^{z}\right)$ are right invertible in $\mathcal{C}(H)$, thus $0 \in \rho_{\text {re }}(T)$. Since $\sigma_{r e}\left(\Delta_{z}(T)\right) \backslash\{0\}=\sigma_{r e}\left(|T|^{z} U|T|^{1-z}\right) \backslash\{0\}=$ $\sigma_{r e}\left(U|T|^{1-z}|T|^{z}\right) \backslash\{0\}=\sigma_{r e}(T) \backslash\{0\}$, we finally get $\sigma_{r e}(T) \subseteq \sigma_{r e}\left(\Delta_{z}(T)\right)$. Let $T$ be a pure coisometry of infinite dimensional null space. Using Proposition 4 of [7, we can see that $\Delta_{z}(T)^{*}=T^{* 2} T$ has an infinite dimensional null space, and hence $0 \in \sigma_{r e}\left(\Delta_{z}(T)\right)$, but clearly $0 \notin \sigma_{r e}(T)$ because $T$ is right invertible. It follows that the inclusion may be strict for all $z \in \overline{\mathcal{S}}$.

(iv) We have seen before ((iii) (a)) that $\lambda I-\Delta_{z}(T) \in \mathcal{F}$ if and only if $\lambda I-T \in \mathcal{F}$. Besides that, we know that $\operatorname{dim}(\mathcal{N}(\lambda I-A B))=\operatorname{dim}(\mathcal{N}(\lambda I-B A))$ for any nonzero $\lambda$, and for any pair $(A, B) \in B(H)$. It leads to $\sigma_{w}\left(\Delta_{z}(T)\right) \backslash\{0\}=\sigma_{w}(T) \backslash\{0\}$. Suppose now that $T$ is a Weyl operator, then we have seen that $U \in \mathcal{F}$ and $|T|^{u} \in \mathcal{F}$ for any $u \in \overline{\mathcal{S}}$ (see (iii) (a)). Then $\Delta_{z}(T) \in \mathcal{F}$ and using the multiplicative property of the index we get $\operatorname{ind}\left(\Delta_{z}(T)\right)=i n d\left(|T|^{z}\right)+i n d(U)+i n d\left(|T|^{1-z}\right)=$ $\operatorname{ind}(T)=0$, so $\Delta_{z}(T)$ is a Weyl operator. In the same manner, we prove that $\Delta_{z}(T)$ is a Weyl operator implies that $T$ is a Weyl operator. Finally, we have obtained the desired equality $\sigma_{w}\left(\Delta_{z}(T)\right)=\sigma_{w}(T)$ for any $z \in \overline{\mathcal{S}}$. 
(v) Recall that $A$ is a Browder operator if and only if $A$ is a Fredholm operator and $z I-A$ is invertible for sufficiently small $z \in \mathbb{C} \backslash\{0\}$ ([13] Theorem 7.9.3). Thus, the desired equality follows from (ii) and (iii) (a).

(vi) As $\sigma_{p}\left(|T|^{z} U|T|^{1-z}\right) \backslash\{0\}=\sigma_{p}\left(U|T|^{1-z}|T|^{z}\right) \backslash\{0\}=\sigma_{p}(T) \backslash\{0\}$, it rests to prove that $0 \in \sigma_{p}(T)$ if and only if $0 \in \sigma_{p}\left(\Delta_{z}(T)\right)$ for any $z \in \overline{\mathcal{S}}$. Assume there exists $x \neq 0$ such that $T x=0$. By Proposition 2.2 (ii), we know that $x \in \mathcal{N}\left(|T|^{z}\right)=$ $\mathcal{N}\left(|T|^{1-z}\right)$, thus $\left.x \in \mathcal{N}\left(\Delta_{z}(T)\right)\right)$. Conversely, suppose that $0 \in \sigma_{p}\left(\Delta_{z}(T)\right)$ and let $x \in[H \backslash\{0\}] \cap \mathcal{N}\left(\Delta_{z}(T)\right)$, then $0=U|T|^{1-z} \Delta_{z}(T) x=T U|T|^{1-z} x$. On the one hand, if $y=U|T|^{1-z} x \neq 0$, we have $\mathcal{N}(T) \neq\{0\}$. On the other hand, $y=0$, using Proposition 2.2 we see that $|T|^{1-z} x \in \mathcal{N}(U)=\mathcal{N}(|T|)=\mathcal{N}\left(|T|^{z}\right)$ and hence $x \in \mathcal{N}(|T|)=\mathcal{N}(T)$. Consideration of both cases leads to $0 \in \sigma_{p}(T)$. This concludes the proof of this assertion.

(vii) Having in view that $\sigma_{\text {app }}(A)=\sigma_{l e}(A) \cup \sigma_{p}(A)$ for each $A \in B(H)$ and using (iii) (b) and (vi), we get $\sigma_{a p p}\left(\Delta_{z}(T)\right)=\sigma_{a p p}(T)$ for any $z \in \overline{\mathcal{S}} \backslash[1+i \mathbb{R}]$ and $\sigma_{\text {app }}\left(\Delta_{z}(T)\right) \subseteq \sigma_{\text {app }}(T)$ when $z \in 1+i \mathbb{R}$. Moreover, let $z \in 1+i \mathbb{R}$ and consider the operator $T$ acting on $l^{2}(\mathbb{N}) \oplus l^{2}(\mathbb{N})$ and defined in (iii) (b). Since $\Delta_{z}(T)$ is an isometry, we clearly have $0 \notin \sigma_{\text {app }}\left(\Delta_{z}(T)\right)$ but $\left\|T\left(e_{n} \oplus 0\right)\right\| \rightarrow 0$ which implies $0 \in \sigma_{\text {app }}(T)$. Therefore the inclusion $\sigma_{\text {app }}\left(\Delta_{z}(T)\right) \subseteq \sigma_{\text {app }}(T)$ may be strict when $z \in 1+i \mathbb{R}$

(viii) We have $\sigma_{\text {res }}\left(|T|^{z} U|T|^{1-z}\right) \backslash\{0\}=\sigma_{\text {res }}\left(U|T|^{1-z}|T|^{z}\right) \backslash\{0\}=\sigma_{\text {res }}(T) \backslash\{0\}$ for each $z \in \overline{\mathcal{S}}$. Let $z=i b \in i \mathbb{R}$, taking into account that $T$ is one-to-one if and only if $\Delta_{z}(T)$ is also (see (vi)), we remark that $\Delta_{z}(T)=|T|^{i b} T|T|^{-i b}$ is unitarily similar to $T$ as soon as $0 \in \sigma_{\text {res }}(T) \cup \sigma_{\text {res }}\left(\Delta_{i b}(T)\right)$. We infer that $0 \in \sigma_{\text {res }}\left(\Delta_{i b}(T)\right)$ if and only if $0 \in \sigma_{\text {res }}(T)$, and hence $\sigma_{\text {res }}\left(\Delta_{i b}(T)\right)=\sigma_{\text {res }}(T)$. From now on, we suppose that $z \in \overline{\mathcal{S}} \backslash i \mathbb{R}]$. Assume that $0 \in \sigma_{\text {res }}\left(\Delta_{z}(T)\right)$, then $\Delta_{z}(T)$ is one-to-one and there exists a non-zero $x \in H$ such that $\Delta_{z}(T)^{*} x=0$. It implies that $|T|^{u}$ is one-to-one for any $u \in \mathcal{P}_{+}$, and hence that $U^{*}|T|^{\bar{z}} x=0$. Obviously, we have $y=|T|^{\bar{z}} x \neq 0$ and $y \in \mathcal{N}\left(U^{*}\right)=\mathcal{N}\left(T^{*}\right)$. Finally, it leads to $0 \in \sigma_{\text {res }}(T)$, thus $\sigma_{\text {res }}\left(\Delta_{z}(T)\right) \subseteq \sigma_{\text {res }}(T)$ for any $\left.z \in \overline{\mathcal{S}} \backslash i \mathbb{R}\right]$.

Now, let $z \in \overline{\mathcal{S}} \backslash i \mathbb{R}], H$ be $L^{2}([0,1] d t), R$ be the positive operator defined by $R(f)(t)=t^{1 / \Re(z)} f(t)$ for any $f \in H$. Consider an isometry $U$ acting on $H$ whose range is $\{\mathbf{1}\}^{\perp}$, and set $T=U R$. We easily check that $T$ is one-to-one and that $T^{*}(\mathbf{1})=0$, so $0 \in \sigma_{\text {res }}(T)$. Observe that $R=|T|$ and that $\mathcal{N}(U)=\{0\}=\mathcal{N}(T)$, it follows that $T=U R$ is exactly the polar decomposition of $T$ and then $\Delta_{z}(T)=$ $R^{z} U R^{1-z}$. Suppose that $0 \in \sigma_{\text {res }}\left(\Delta_{z}(T)\right)$, then there exists a non-zero $f \in H$ such that $0=R^{1-\bar{z}} U^{*} R^{\bar{z}}(f)$, it implies that $R^{\bar{z}}(f) \in \mathcal{N}\left(U^{*}\right)=\mathbb{C} 1$. So $R^{\bar{z}}(f)$ is almost everywhere equal to a non-zero constant $a$. It forces the function $\psi: t \mapsto a t^{-1}$ to be in $L^{2}([0,1] d t)$, a fact which is absurd. Consequently, in this case, the inclusion $\sigma_{\text {res }}\left(\Delta_{z}(T)\right) \subseteq \sigma_{\text {res }}(T)$ is strict for any $z \in \overline{\mathcal{S}} \backslash[i \mathbb{R}]$. This completes the proof of Theorem 4.1 . 
Remark 4.2. Note that we have proved in passing (see (iv)) that if $T$ is a Fredholm operator, then $\Delta_{z}(T) \in \mathcal{F}$ for any $z \in \overline{\mathcal{S}}$ and the index is constant on the Aluthge operator field $\left(\Delta_{z}(T)\right)_{z \in \overline{\mathcal{S}}}$.

4.0.2. Generalized Yamazaki formula. Let $T \in B(H)$ and denote by $r(T)$ its spectral radius. Recall the famous Gelfand formula

$$
r(T)=\lim \left\|T^{n}\right\|^{\frac{1}{n}}=\inf _{n \geqslant 0}\left\|T^{n}\right\|^{\frac{1}{n}} .
$$

In [23], T. Yamazaki gives an alternative formula involving the Aluthge transformation $\Delta_{1 / 2}$ :

$$
r(T)=\lim \left\|\Delta_{\frac{1}{2}}^{n}(T)\right\|=\inf _{n \geqslant 0}\left\|\Delta_{\frac{1}{2}}^{n}(T)\right\| .
$$

Notice that a simpler proof is given in [2]. See also [8] for other spectral radius formulas via Aluthge transformation. In [3], the authors proved that for any square matrix $M$ and any $\alpha \in] 0,1[$ we have

$$
r(M)=\lim \left\|\Delta_{\alpha}^{n}(M)\right\|=\inf _{n \geqslant 0}\left\|\Delta_{\alpha}^{n}(M)\right\| .
$$

In [21, it is proved that Formula 4.1 holds if we replace $M$ by any invertible operator acting on an infinite dimensional Hilbert space. In the next theorem, we show that we can drop the assumption that $T$ is invertible and we can consider $\Delta_{z}$ for any $z \in \mathcal{S}$.

Theorem 4.3. Let $T$ be a operator acting on a Hilbert space $H$, then we have

$$
r(T)=\lim \left\|\Delta_{z}^{n}(T)\right\|=\inf _{n \geqslant 0}\left\|\Delta_{z}^{n}(T)\right\|
$$

for any $z \in \mathcal{S}$.

Proof. Firstly, from Proposition 4.1 we know that $\sigma\left(\Delta_{z}^{n}(T)\right)=\sigma(T)$, so $r(T)=$ $r\left(\Delta_{z}^{n}(T)\right) \leqslant\left\|\Delta_{z}^{n}(T)\right\|$ and hence $r(T) \leqslant \inf _{n \geqslant 0}\left\|\Delta_{z}^{n}(T)\right\|$. Thus from now on, by homogeneity we may assume that $\inf _{n \geqslant 0}\left\|\Delta_{z}^{n}(T)\right\|=1$. We set $z=a+i b$ with $a \in] 0,1$ [. Let $A, B$ be two positive operators acting on $H$ and $X \in B(H)$. Recall that Heinz inequality (see [14]) tells us that

$$
\left\|A^{r} X B^{1-r}\right\| \leqslant\|A X\|^{r}\|X B\|^{1-r}
$$

for each $r \in[0,1]$. Using Heinz inequality, for any $m \geqslant 1$ we get

$$
\begin{gathered}
\left\|\Delta_{z}(T)^{m}\right\|=\left\||T|^{z} T^{m-1} U|T|^{1-z}\right\| \leqslant\left\||T|^{a} T^{m-1} U|T|^{1-a}\right\| \\
\leqslant\left\||T| T^{m-1} U\right\|^{a}\left\|T^{m-1} U|T|\right\|^{1-a} \leqslant\left\|T^{m}\right\|^{a}\left\|T^{m}\right\|^{1-a}=\left\|T^{m}\right\| .
\end{gathered}
$$

By (4.2), we see that the sequence $\left(\left\|\Delta_{z}^{n}(T)^{m}\right\|\right)_{n \geqslant 0}$ is decreasing for any $m \in \mathbb{N}^{*}$. On the one hand, it implies that the operator $R=\Phi\left(\left(\Delta_{z}^{n}(T)\right)_{n \geqslant 0}\right)$, acting on the ultrapower of $\mathcal{H}$ associated with a Banach limit $\mathcal{L}$, is well defined. On the other hand, by Proposition 2.3 (i) and by 4.2 we get

$$
\left\|R^{m}\right\|=\lim _{n \rightarrow+\infty}\left\|\Delta_{z}^{n}(T)^{m}\right\| \leqslant\left\|T^{m}\right\|
$$

a fact which leads to $r(R) \leqslant r(T)$. Let $m \geqslant 1$, observe that $\left\|\Delta_{z}^{m}(R)\right\|=$ $\lim \left(\left\|\Delta_{z}^{m+n}(T)\right\|\right)=\inf _{n \geqslant 0}\left\|\Delta_{z}^{n}(T)\right\|=1=\|R\|$. Applying Theorem 2.5 (ii), we obtain that $\left\|R^{m+1}\right\|=1$. It follows that $1=r(R) \leqslant r(T)$ and finally that $r(T)=1$. It ends the proof of Theorem 4.3 . 
Remark 4.4. Let $T \in B(H)$ be a one-to-one operator with dense range such that $r(T)<\|T\|$. In this case, we have

$$
\left\|\Delta_{1+i t}(T)\right\|=\||T| U\|=\||T|\|=\|T\|=\left\|\Delta_{i t}(T)\right\|
$$

for any $t \in \mathbb{R}$. If $T$ is one-to-one with dense range, then $\Delta_{z}(T)$ is still one-to-one with dense range. So, by iteration we obtain $\left\|\Delta_{1+i t}^{n}(T)\right\|=\|T\|=\left\|\Delta_{i t}^{n}(T)\right\|$ for each $n \in \mathbb{N}$. Therefore, a Yamazaki type formula is not true in general when $z$ belongs to the boundary of $\mathcal{S}$.

\section{REFERENCES}

1. A. Aluthge, On p-hyponormal operator for $0<p<1$, Integral Equations and Operator Theory, 13 (1990), 307-315.

2. T. Ando, Aluthge transforms and the convex hull of the spectrum of a Hilbert space operator, Operator Theory: Advances and Applications, Vol. 160 (2005), 21-39.

3. J. Antezana, P. Massey, D. Stajanoff, $\lambda$-Aluthge transforms and Schatten ideals, Linear Algebra and its Applications, 405 (2005), 177-199.

4. C. Benhida, R. E. Curto, S. H. Sang, and J. Yoon Joint spectra of spherical Aluthge transforms of commuting n-tuples of Hilbert space operators, C. R. Math. Acad. Sci. Paris, 357 (2019), 799-802.

5. R. Ben Taher and M. Rachidi, 2-Variable weighted shifts and recursiveness : toral Aluthge transform and subnormality, The Journal of Analysis (2020).

6. S. K. Berberian, Approximate proper vectors, Proc. Amer. Math. Soc., 13 (1962), no. 3, 111-114.

7. G. Cassier and J. Verliat, Stability for some operator classes by Aluthge transform, Operator Theory Live, Theta 2010, 51-67.

8. F. Chabbabi and M. Mbekhta, New formulas for the spectral radius via $\lambda$-Aluthge transform, Linear Algebra Appl. 515 (2017), 246-254.

9. R. E. Curto, J. Kim, and J. Yoon The Aluthge transform of unilateral weighted shifts and the square root problem for finitely atomic measures, Math. Nachr. 292 (2019), no. 11, 2352-2368.

10. R. E. Curto and J. Yoon Aluthge transforms of 2-variable weighted shifts, Integral Equations Operator Theory 90 (2018), no. 5, Article: 52.

11. P.A. Fillmore, J.G. Stampfli, and J.P. Williams, On the essential numerical range, the essential spectrum, and a problem of Halmos, Acta Szeged 33 (1972), 179-192.

12. C. Foias, I. B. Jung, E. Ko and C. Pearcy, Complete contractivity of maps associated with the Aluthge and Duggal transforms, Pacific Journal of Mathematics Vol. 209 (2003), 249-259.

13. R. E. Harte, Invertibility and singularity, Dekker New York, 1988.

14. E. Heinz, Beiträge zur Störungstheorie der Spektalzerleung, Math. Ann. 123 (1951), 415438.

15. K. Hoffman, Banach spaces of analytic functions, Prentice-Hall Series in Modern Analysis, Prentice Hall Inc., Englewood Cliffs, N. J., 1962.

16. S. Hildebrandt, Über den numerishen Wertebereich eines Operators, Math. Ann. 163 (1966), 230-247.

17. T. Huruya, A note on p-hyponormal operators, Proc. Amer. Math. Soc. 125 (1997), 36173624 .

18. I. B. Jung, E. Ko and C. Pearcy, Aluthge transforms of operators, Integral Equations Operator Theory 37 (2000), 437-448.

19. M. Mbekhta, Conorme et inverse généralisé dans les $C^{*}$-algèbres, Canad. Math. Bull.Vol. 35 (1992) no. 4, 515-522.

20. K. Okubo, On weakly unitarily invariant norms and Aluthge transformation, Linear Algebra and its Applications 371 (2003), 369-375. 
21. T. Tam, $\lambda$-Aluthge Iteration and Spectral Radius, Integral Equations and Operator Theory 60 (2008), 591-596.

22. P. Y. Wu, Numerical range of Aluthge transform of operators, Linear Algebra and its Applications 357 (2002), 295-298.

23. T. Yamazaki, On numerical range of the Aluthge transformation, Linear Algebra and its Applications 341 (2002), 111-117.

24. T. Yamazaki, An expression of spectral radius via Aluthge transformation, Proc. Amer. Math. Soc. 130 (2002), 1131-1137.

1 Université de Lyon 1; Institut Camille Jordan CNRS UMR 5208; 43, BouleVARD Du 11 Novembre 1918, F-69622 Villeurbanne, France.

Email address: cassier@math.univ-lyon1.fr

2 École normale supérieure Paris-Saclay,61 Avenue du Président Wilson, 94230 Cachan, France

Email address: thomas.perrin@ens-paris-saclay.fr 